\title{
RADIAL VELOCITY VARIABILITY OF FIELD BROWN DWARFS
}

\author{
L. Prato ${ }^{1}$, G. N. Mace ${ }^{2}$, E. L. Rice ${ }^{3,4}$, I. S. McLean ${ }^{5}$, J. Davy KirkPatrick ${ }^{6}$, A. J. Burgasser ${ }^{7}$, And Sungsoo S. Kim ${ }^{8}$ \\ ${ }^{1}$ Lowell Observatory, 1400 West Mars Hill, Road, Flagstaff, AZ 86001, USA; lprato@lowell.edu \\ ${ }^{2}$ Department of Astronomy, University of Texas, R.L. Moore Hall, Austin, TX 78712, USA \\ ${ }^{3}$ Department of Engineering Science and Physics, College of Staten Island, 2800 Victory Boulevard, Staten Island, NY 10314, USA \\ ${ }^{4}$ Department of Astrophysics, American Museum of Natural History, Central Park West at 79th Street, New York, NY 10024, USA \\ 5 Department of Physics and Astronomy, UCLA, 430 Portola Plaza, Box 951547, Los Angeles, CA 90095-1547, USA \\ ${ }^{6}$ Infrared Processing and Analysis Center, California Institute of Technology, Pasadena, CA 91125, USA \\ ${ }^{7}$ Center for Astrophysics and Space Science, University of California San Diego, La Jolla, CA 92093, USA \\ ${ }^{8}$ Department of Astronomy \& Space Science, Kyung Hee University, Yongin, Kyungki 446-701, Korea \\ Received 2015 February 11; accepted 2015 June 6; published 2015 July 14
}

\begin{abstract}
We present paper six of the NIRSPEC Brown Dwarf Spectroscopic Survey, an analysis of multi-epoch, highresolution $(R \sim 20,000)$ spectra of 25 field dwarf systems (3 late-type M dwarfs, $16 \mathrm{~L}$ dwarfs, and $6 \mathrm{~T}$ dwarfs) taken with the NIRSPEC infrared spectrograph at the W. M. Keck Observatory. With a radial velocity (RV) precision of $\sim 2 \mathrm{~km} \mathrm{~s}^{-1}$, we are sensitive to brown dwarf companions in orbits with periods of a few years or less given a mass ratio of 0.5 or greater. We do not detect any spectroscopic binary brown dwarfs in the sample. Given our target properties, and the frequency and cadence of observations, we use a Monte Carlo simulation to determine the detection probability of our sample. Even with a null detection result, our $1 \sigma$ upper limit for very low mass binary frequency is $18 \%$. Our targets included seven known, wide brown dwarf binary systems. No significant RV variability was measured in our multi-epoch observations of these systems, even for those pairs for which our data spanned a significant fraction of the orbital period. Specialized techniques are required to reach the high precisions sensitive to motion in orbits of very low-mass systems. For eight objects, including six T dwarfs, we present the first published high-resolution spectra, many with high signal to noise, that will provide valuable comparison data for models of brown dwarf atmospheres.
\end{abstract}

Key words: binaries: spectroscopic - brown dwarfs - techniques: spectroscopic

\section{INTRODUCTION}

Star and planet formation processes both give rise to objects in the $\sim 1$ to $20 M_{\text {Jup }}$ range (e.g., Luhman et al. 2005; Bakos et al. 2009; Marois et al. 2010; Joergens et al. 2013). Naively, objects of higher mass are typically assumed to form primarily via the star formation process and objects of lower mass are assumed to form primarily from a proto-planetary disk. This simplification is directly testable with a variety of approaches, both theoretical and observational. One straightforward observational approach is the study of multiplicity: do brown dwarfs come in pairs as frequently and with the same binary properties as stars? Although trends are suggestive among stellar visual binaries, i.e., decreasing frequency with later spectral types (e.g., Siegler et al. 2003), the frequency of short-period brown dwarf binaries is relatively uncertain (Burgasser et al. 2012).

Binary parameter space is multi-dimensional. For a given spectral type, binaries may be examined in terms of companion frequency, separation, mass ratio distribution, or secondary mass distribution. Mazeh et al. (2003), for example, showed that for main sequence $\mathrm{M}$ stars the secondary mass distribution does not conform to a standard initial mass function but instead follows a relatively flat distribution for a primary sample with $M \sim 0.7 \pm 0.1 M_{\odot}$ and orbital period $P<3000$ days. This represents one small slice of a parameter space that may also be studied in diverse populations: young, intermediate age, old, metal-poor, clustered, etc. Specific comparisons between these samples provide a wealth of diagnostics for understanding the similarities and differences in formation and evolution of distinct groups of objects.

In a sample of 454 F6-K3 solar-type stellar systems within $25 \mathrm{pc}$ of the Sun, Raghavan et al. (2010) found a total fraction of binaries and higher order multiples of $44 \% .{ }^{9}$ Among other results, they reconfirm the existence of the brown dwarf desert, the pronounced dearth of brown dwarf mass (i.e., $\sim 10-80$ $M_{\text {Jup }}$ ) companions to stars in orbits with periods less than a few years (e.g., Grether \& Lineweaver 2006; Metchev \& Hillenbrand 2009).

Is the brown dwarf desert the result of dynamical evolution preferentially impacting lower mass companions (e.g., Reipurth \& Clarke 2001; Armitage \& Bonnell 2002) or does it have more to do with poorly understood barriers to the formation of tightly bound companions of brown dwarf mass? In a radial velocity $(\mathrm{RV})$ survey with a few hundred $\mathrm{m} \mathrm{s}^{-1}$ precision of $>100$ stars in the Taurus star forming region (Crockett et al. 2012), no brown dwarf companions to 1-3 Myr old stars have been observed (N. I. Mahmud et al. 2015, in preparation), indicating that the existence of the desert is more likely related to formation than dynamical evolution in origin. Among 1-10 Myr old populations, to date only one brown dwarf-brown dwarf short-period ( $\lesssim 1$ year) spectroscopic binary pair has been identified (Stassun et al. 2006). Joergens et al. (2010) found an orbital period of 5.2 years for the young Chameleon brown dwarf binary Cha $\mathrm{H} \alpha 8$ and Joergens (2008) estimates a period of $>12$ years for the pair CHXR74. However, the results of Stassun et al. and Joergens are based, respectively, on a survey for eclipsing systems and on a relatively small sample and thus are likely incomplete. Joergens estimates a binary frequency among very low mass young objects of $10_{-8}^{+18} \%$.

\footnotetext{
9 Thus, although the majority of these systems may not be multiple, the majority of the stars studied reside in multiple systems, as previously concluded (e.g., Abt \& Levy 1976; Duquennoy \& Mayor 1991).
} 
Among intermediate age brown dwarf spectroscopic binaries, Basri \& Martín (1999) found the first brown dwarf pair, PPL 15, a 5.8 day period system, in a study of the Pleiades. Simon et al. (2006) studied the $100 \mathrm{Myr}$ old brown dwarf binary GL 569B (Zapatero Osorio et al. 2004), a pair with a $\sim 2.4$ year orbit, a semimajor axis of 0.89 AU. They detected some evidence for a third spectroscopic component in the system, yet to be confirmed.

RV surveys among field brown dwarfs, sensitive to binaries with periods of several years or less and semimajor axes of a few AU, have yielded a handful of definitive detections. In a sample of 59 field brown dwarfs, Blake et al. (2010) found a tight binary $(a<1 \mathrm{AU})$ frequency of $2.5_{-1.6}^{+8.6 \%}$. They had previously identified and measured the orbit of the 247 day period system 2MASS 0320-04 (Blake et al. 2008), independently identified on the basis of spectral analysis by Burgasser et al. (2008), combined their RV measurements with the astrometry of Dahn et al. (2008) for the $\sim 607$ day period system LSR J1610-0040, and present RV data on two wide substellar pairs with periods of $>10$ years (2MASS J150747691627386 and 2MASS J07464256+2000321). Zapatero Osorio et al. (2007) measured space motions for over a dozen field brown dwarfs but found no spectroscopic pairs in their sample. Burgasser et al. (2012) presented a solution for the spectroscopic orbit of the 148 day period pair SDSS J000649.16$085246.3 \mathrm{AB}$, in common proper motion with the very low mass star LP 704-48, with M9 and T0 components straddling the substellar limit. Although Basri \& Reiners (2006) indicate an overall spectroscopic binary fraction for field brown dwarfs and very low mass stars of $11 \%$ in their RV survey of 53 targets, only three L dwarfs in their sample show some level of RV variability. Of these three, 2MASS J15065441+1321060 was subsequently shown by Blake et al. to be non-variable and 2MASS J15074769-1627386 and 2MASS J07464256 +2000321 are long-period systems identified as binaries with imaging observations. Other brown dwarf pairs have been identified with imaging (e.g., Lodieu et al. 2007), microlensing (e.g., Bennett et al. 2008), and astrometry (e.g., Sahlmann et al. 2013). Spectral brown dwarf binaries, systems that appear single in light of existing data but are spectroscopically peculiar, implicating the possible presence of a companion, may be numerous. Bardalez Gagliuffi et al. (2014) have identified 50 candidates, although Artigau et al. (2009) and Radigan et al. (2013) identified cases in which the brown dwarf binary candidates were instead found to have heterogeneous cloud covers. Thus it is unlikely that all spectral binaries have $<1$ year orbits, but two have been confirmed as such to date (Blake et al. 2008; Burgasser et al. 2012).

No short period (<100 days) field brown dwarf spectroscopic binaries are known, but one intermediate age and one young system with periods of just a few days were identified by Basri \& Martín (1999) and Stassun et al. (2006), respectively. Short period systems ought to be the most straightforward to identify; however, RV surveys for brown dwarf multiples require the world's biggest telescopes and generous time allocations, challenging to obtain, and are fraught with bias. Yet without such work our understanding of substellar multiplicity is skewed toward the anecdotal, and astronomers' grasp of the basis for planetary mass companion formation is isolated from the context of brown dwarf and stellar mass companion formation.
We report here on 11 years of dynamical observations of over two dozen field brown dwarfs taken at high spectral resolution in the near-infrared (IR) at the Keck II telescope. The intrinsic faintness of brown dwarfs, particularly the late $\mathrm{L}$ and $\mathrm{T}$ types, presents a challenge to high-resolution spectroscopic observations. However, this is the only method by which we may derive the RV data necessary for calculating space motions, and hence possible moving group or cluster membership, and the telltale RV variability of a short-period binary. Measurements of $v \sin i$ provide a lower limit on rotational velocity, crucial for understanding angular momentum evolution. Ultimately, with sufficiently precise data, the combination of RV versus phase together with the angularly resolved orbits for the few year or few tens of year period systems will yield the absolute component masses of brown dwarfs in binaries, invaluable for furthering our understanding of brown dwarf structure and evolution (Dupuy et al. 2010; Konopacky et al. 2010). Because brown dwarfs emit the bulk of their energy at wavelengths greater than $\sim 1 \mu \mathrm{m}$, IR spectroscopy provides the best approach for their RV measurements.

Our goals were to identify brown dwarfs in spectroscopic binary systems and to measure the dynamical properties of any such pairs discovered. This project to identify short-period brown dwarf multiples is the latest contribution to the NIRSPEC Brown Dwarf Spectroscopic Survey (BDSS; McLean et al. 2001, 2003, 2007; McGovern et al. 2004; Rice et al. 2010) and leverages over a decade of observations to characterize brown dwarfs at high spectral resolution. We find that a critical factor in a productive survey hinges on the RV precision; sensitivity to RV variability scales rapidly with this parameter. We describe our sample, observations, and data reduction in Section 2 and discuss our data analysis in Section 3. Section 4 provides a discussion of our results and we briefly summarize our work in Section 5.

\section{OBSERVATIONS AND DATA REDUCTION}

Targets were selected for a range of spectral types across the span of late M, L, and T dwarfs and on the basis of magnitude $(J \lesssim 15 \mathrm{mag})$ and accessibility from the Keck Observatory $(\delta \gtrsim$ $\left.-30^{\circ}\right)$. The complete target list and observing log appears in Table 1 which lists the object name (column 1), R.A. and decl. (columns 2 and 3), spectral type (column 4), 2MASS $J$ magnitude (column 5), reference for discovery paper (column 6 ), and the UT dates of observation (column 7).

Observations were carried out with the high-resolution, cross-dispersed echelle mode of the facility, near-IR, cryogenic spectrograph NIRSPEC (McLean et al. 1998, 2000) on the Keck II 10 m telescope on Mauna Kea, Hawaii. The NIRSPEC science detector is a $1024 \times 1024$ pixel ALADDIN InSb array; a $256 \times 256$ pixel $\mathrm{HgCdTe}$ array in a slit viewing camera was used for source acquisition. The N3 (J-band) filter with the $12 \times 0^{\prime \prime} .432$ (3 pixel) slit, an echelle angle of 63.00 , and a grating angle of 34.08 produces a resolving power of $R=\lambda / \Delta$ $\lambda \approx 20,000$ and nearly continuous coverage from 1.165 to $1.324 \mu \mathrm{m}$ (orders 58-65; McLean et al. 2007). Observations made on 2000 July 25 and 29 employed the $12 \times 0$ ".576 (4 pixel) slit, yielding a resolution of $\sim 15,000$. Internal whitelight spectra, dark frames, and arc lamp spectra were obtained for flat-fielding, dark current correction, and wavelength calibration. Science exposures were made in $600 \mathrm{~s}$ nodded $\mathrm{AB}$ pairs at two locations along the slit. 
Table 1

Target List and Observing Log

\begin{tabular}{|c|c|c|c|c|c|c|}
\hline $\begin{array}{l}\text { Object } \\
\text { (1) }\end{array}$ & $\begin{array}{c}\alpha \\
(\mathbf{J} 2000.0) \\
(2)\end{array}$ & $\begin{array}{c}\delta \\
(\mathrm{J} 2000.0) \\
(3)\end{array}$ & $\begin{array}{l}\text { Spectral } \\
\text { Type }^{\mathrm{a}} \\
(4)\end{array}$ & $\begin{array}{c}J \\
(\mathrm{mag}) \\
(5)\end{array}$ & $\begin{array}{l}\text { Discovery } \\
\text { Reference } \\
\quad(6)\end{array}$ & $\begin{array}{l}\text { UT Dates } \\
\text { of Observation } \\
\text { (7) }\end{array}$ \\
\hline GJ 406 (Wolf 359) & 105628.9 & 070053 & $\mathrm{M}_{o} 6$ & $7.09 \pm 0.02$ & $(1)$ & 2002 Apr 23, 2006 Jan 11, May 20 \\
\hline 2 MASS J22344161+4041387 & 223441.6 & 404139 & $\mathrm{M}_{o} 6$ & $12.57 \pm 0.02$ & (2) & 2006 Oct 6,2007 May 29, 2009 Nov 8 \\
\hline 2MASS J01400263+2701505 & 014002.6 & 270151 & $\mathrm{M}_{o} 8.5$ & $12.49 \pm 0.02$ & (3) & 2000 Dec 4, Dec 6, 2002 June 23 \\
\hline 2MASP J0345432+254023 & 034543.2 & 254023 & L1 & $14.00 \pm 0.03$ & (4) & 2000 Dec 4, Dec 6, 2006 Jan 11 \\
\hline 2MASS J07464256+2000321 ${ }^{\mathrm{b}}$ & 074642.6 & 200032 & $\mathrm{~L} 1$ & $11.76 \pm 0.02$ & $(5)$ & 2002 Jan 1, 2006 Jan 10 \\
\hline 2MASS J02081833+2542533 & 020818.3 & 254253 & $\mathrm{~L}_{o} 1$ & $13.99 \pm 0.03$ & (6) & 2000 Jul 25, Jul 29, 2008 Dec 6 \\
\hline 2MASS J16580380+7027015 & 165803.8 & 702702 & $\mathrm{~L}_{o} 1$ & $13.29 \pm 0.02$ & (3) & 2000 Jul 25, 2007 May 29 \\
\hline 2MASS J20575409-0252302 & 205754.1 & -025230 & L1.5 & $13.12 \pm 0.02$ & (2) & 2000 Jul 25, 2003 Jul 20, 2007 May 29 \\
\hline 2MASS J00154476+3516026 & 001544.8 & 351603 & $\mathrm{~L}_{o} 2$ & $13.87 \pm 0.03$ & (6) & 2000 Jul 25, 2008 Dec 5 \\
\hline Kelu- $1^{\mathrm{b}}$ & 130540.2 & -254106 & $\mathrm{~L}_{o} 2$ & $13.41 \pm 0.03$ & (7) & 2002 Apr 23, 2003 May 12, 2006 Jan 10, May 19, 2007 May 31, 2008 Mar 20, 2011 Feb 9 \\
\hline 2MASS J21041491-1037369 & 210414.9 & -103737 & $\mathrm{~L}_{o} 2.5$ & $13.84 \pm 0.03$ & (2) & 2007 May 30, 2009 Nov 8 \\
\hline G196-3B & 100420.7 & 502300 & $\mathrm{~L}_{o} 3 \beta^{\mathrm{d}}$ & $14.83 \pm 0.05$ & $(8)$ & 2002 Apr 23, 2006 Jan 11, May 20, 2008 Mar 21 \\
\hline 2MASS J15065441+1321060 & 150654.4 & 132106 & $\mathrm{~L}_{o} 3$ & $13.37 \pm 0.02$ & (3) & 2000 Jul 25, Jul 28, 2007 May 30, 2008 Mar 21 \\
\hline GD165B & 142439.1 & 091710 & L3 & $15.69 \pm 0.08$ & (9) & 2002 Apr 23, 2003 May 13, 2006 May 20, 2011 Feb 5, 9 \\
\hline 2MASS J22244381-0158521 & 222443.8 & -015852 & L3.5 & $14.07 \pm 0.03$ & (6) & 2000 Jul 25, 2007 May 30 \\
\hline 2MASS J00361617+1821104 & 003616.2 & 182110 & L4 & $12.47 \pm 0.03$ & $(5)$ & 2000 Jul 25, Jul 29, Dec 4, Dec 5, Dec 6, 2001 Dec 30, 2005 Dec 11 \\
\hline 2MASS J15074769-1627386 ${ }^{\mathrm{c}}$ & 150747.7 & -162739 & L5.5 & $12.83 \pm 0.03$ & $(5)$ & 2000 Apr 25, Jul 25, Jul 28, 2007 May 29, 2008 Mar 21 \\
\hline DENIS-P J0205.4-1159 & 020529.4 & -115930 & L5.5 & $14.59 \pm 0.03$ & (10) & 2001 Oct 9, Dec 29, 2006 Jan 10 \\
\hline DENIS-P J1228.2-1547 & 122815.2 & -154735 & L6 & $14.38 \pm 0.03$ & $(10)$ & 2007 May 30, 2011 Feb 5, June 8 \\
\hline SDSSp J042348.57-041403.5 & 042348.6 & -041404 & T0 & $14.47 \pm 0.03$ & (11) & 2001 Oct 9, 2005 Dec 11, 2006 Jan 10 \\
\hline SDSSp J125453.90-012247.4 & 125453.9 & -012247 & $\mathrm{~T} 2$ & $14.89 \pm 0.04$ & (12) & 2003 May 14, 2007 May 31 \\
\hline 2MASS J22541892+3123498 & 225418.9 & 312350 & $\mathrm{~T} 4$ & $15.26 \pm 0.05$ & (13) & 2003 Aug 10, 2005 Jul 19 \\
\hline 2MASS J05591914-1404488 & 055919.1 & -140449 & $\mathrm{~T} 4.5$ & $13.80 \pm 0.02$ & (14) & 2001 Oct 9, Dec 29, 2006 Jan 11, 2008 Mar 19 \\
\hline 2MASS J23565477-1553111 & 235654.8 & -155311 & T5.5 & $15.82 \pm 0.06$ & (13) & 2005 Jul 19, Dec 10 \\
\hline 2MASS J09373487+2931409 & 093734.9 & 293141 & T6p & $14.65 \pm 0.04$ & $(13)$ & 2002 Apr 23, 2003 Mar 24, May 12, 2006 Jan 10, May 19 \\
\hline
\end{tabular}

Notes.

${ }^{a}$ Spectral types are from DwarfArchives.org; IR type is provided unless only optical type is available, indicated with ${ }_{o}$ subscript.

${ }^{\mathrm{b}}$ Binary system.

RV variable according to Blake et al. (2010).

${ }^{\mathrm{d}}$ The $\beta$ designation indicates intermediate surface gravity (Cruz et al. 2009).

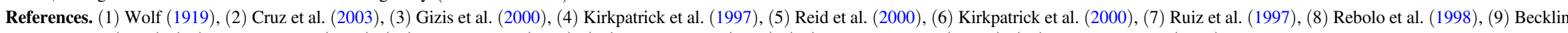
\& Zuckerman (1988), (10) Delfosse et al. (1997), (11) Geballe et al. (2002), (12) Leggett et al. (2000), (13) Burgasser et al. (2002b), (14) Burgasser et al. (2000). 


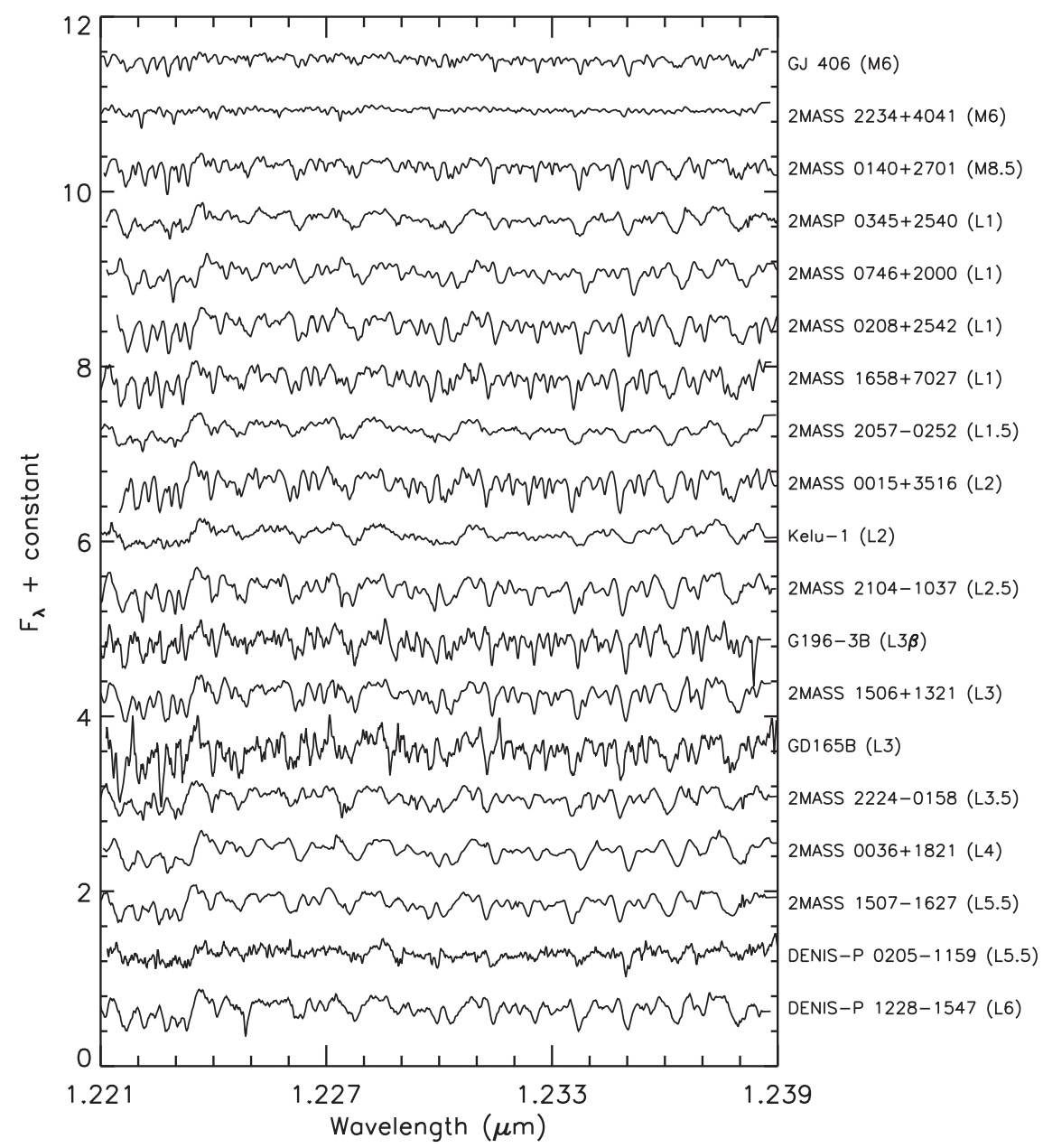

Figure 1. NIRSPEC echelle order $62 \mathrm{M}$ and L dwarf spectral sequence for BDSS RV targets. Spectra are corrected for barycentric velocity, normalized, boxcar smoothed to a 3 pixel resolution element, and offset by a constant.

All spectroscopic reductions were made using the REDSPEC package, software produced at UCLA by S. Kim, L. Prato, and I. McLean specifically for the analysis of NIRSPEC data ${ }^{10}$ as described in McLean et al. (2007).Wavelength solutions were determined using the $\mathrm{OH}$ night sky emission lines in each order; 4-5 OH lines across each of the orders used yielded wavelength solutions with typical uncertainties of better than $0.4 \mathrm{~km} \mathrm{~s}^{-1}$. The two spectral orders most favorable for the analysis, 62 for the L dwarfs (1.221-1.239 $\mu \mathrm{m}$; Figure 1) and 59 for the T dwarfs (1.283-1.302 $\mu \mathrm{m}$; Figure 2), were selected independently on the basis of the presence of deep inherent lines in the brown dwarf targets. Furthermore, an additional advantage of these particular orders is the absence of terrestrial absorption lines, thus avoiding the necessity of division by a featureless telluric standard star. This provided the optimal approach for several reasons: (1) eliminating division by telluric standards maximized the signal-to-noise ratio $(\mathrm{S} / \mathrm{N})$ and avoided the possible introduction of slightly offset spectra and potential small shifts in the brown dwarf absorption lines and hence RV measurements, (2) focusing on the narrowest and deepest lines available yielded the highest possible RV precision; although the KI lines in orders 61 and 65 are deep (e.g., McLean et al. 2007), their breadth is unfavorable to precision RV measurements through cross-correlation, and (3)

\footnotetext{
${ }^{10}$ See http://www2.keck.hawaii.edu/inst/nirspec/redspec.html.
}

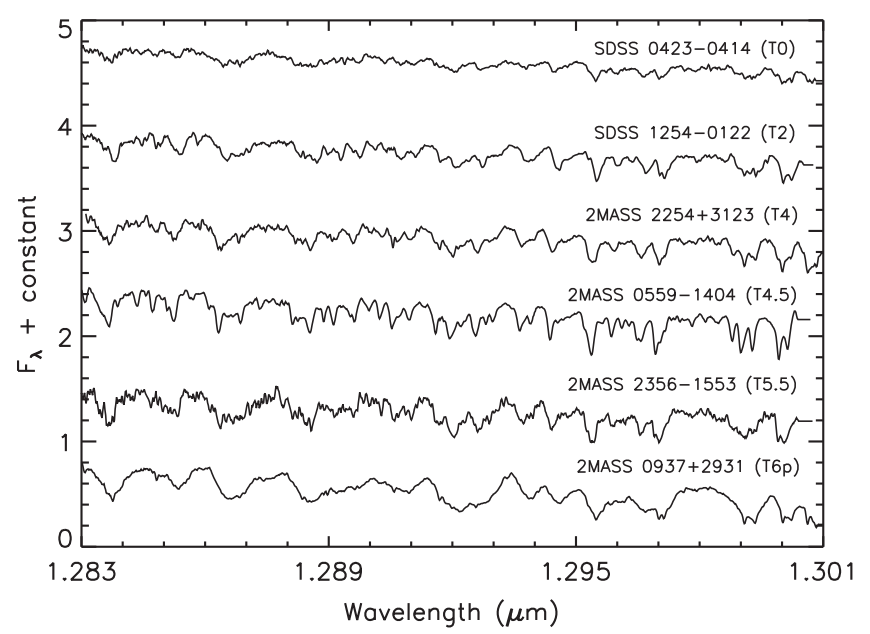

Figure 2. NIRSPEC echelle order $59 \mathrm{~T}$ dwarf spectral sequence for BDSS RV targets. Spectra are corrected for barycentric velocity, normalized, boxcar smoothed to a 3 pixel resolution element, and offset by a constant.

selecting orders 62 and 59 further guaranteed the best possible $\mathrm{RV}$ precision given the regular spacing of the $\mathrm{OH}$ night sky emission lines across both of these orders, required for a superior dispersion solution; this condition was not met for all orders in our $\mathrm{J}$ band setting. Multiple-epoch sequences for the 


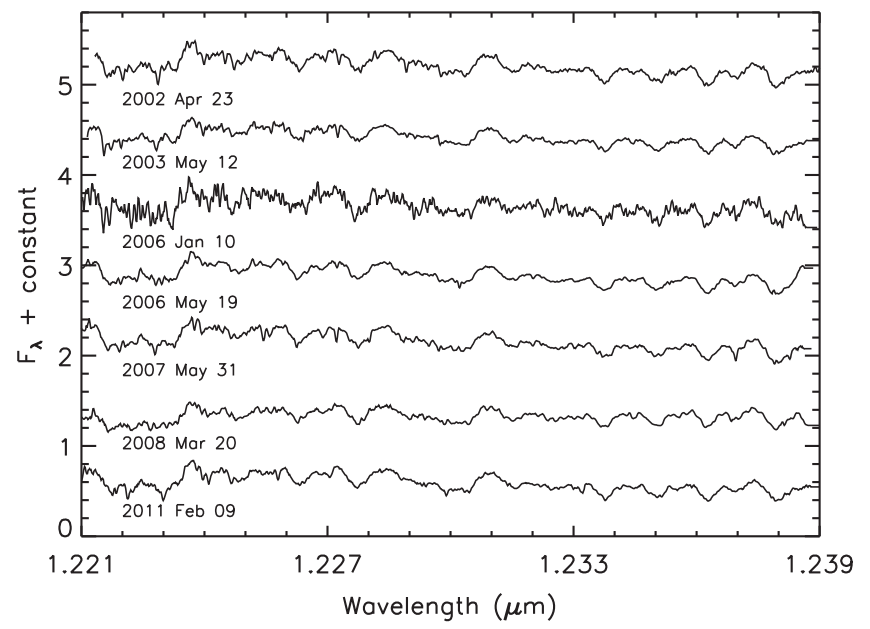

Figure 3. Multiple-epoch spectra for NIRSPEC echelle order 62 of the visual binary Kelu-1. Spectra are corrected and shifted as in Figures 1 and 2. We did not measure any significant RV shift over the nearly nine year baseline of the observations (Section 4.2.3).

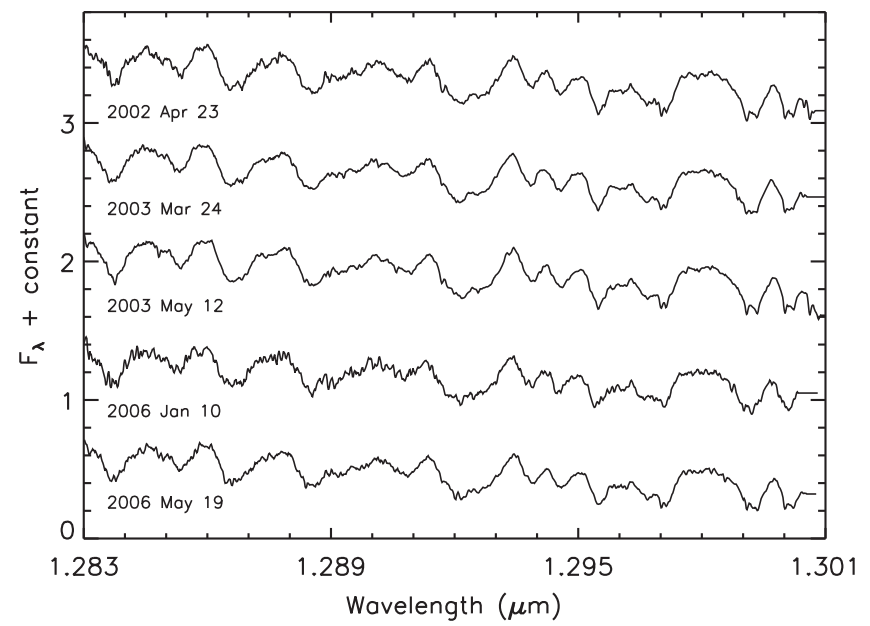

Figure 4. Multiple-epoch spectra for NIRSPEC echelle order 59 of the peculiar T6 dwarf 2MASS 0937+2931. Spectra are corrected and shifted as in Figures 1 and 2. No significant RV shift or line variability was observed in these spectra.

L2 dwarf Kelu-1 and the peculiar T6p dwarf 2M0937 are shown in Figures 3 and 4, respectively.

\section{ANALYSIS}

\subsection{Radial Velocities}

Rice et al. (2010) found typical systematic RV uncertainties of $1-2 \mathrm{~km} \mathrm{~s}^{-1}$ for a sample observed with a similar methodology, similar signal to noise, and with some overlap in target data with this paper (Table 2). We thus adopt a conservative $2 \mathrm{~km} \mathrm{~s}^{-1}$ internal uncertainty for our RVs. We tested this estimate by cross-correlation of the RV invariant target 2M0036 (Blake et al. 2010), an L4 dwarf, for which we obtained 7 epochs over more than 5 years. The maximum RV shift between epochs was $1.91 \mathrm{~km} \mathrm{~s}^{-1}$; the standard deviation in the RV shift for all epochs was $0.59 \mathrm{~km} \mathrm{~s}^{-1}$. Thus $2 \mathrm{~km} \mathrm{~s}^{-1}$ provides a reasonable if conservative internal uncertainty on individual RV measurements.

At least two, and as many as seven, spectra were taken for each of our targets. We tested for RV variability by crosscorrelating the highest signal-to-noise spectrum against the spectra from all other epochs for a given target; no significant variability was detected (Section 4). Table 2 lists the number of spectra taken for each object (column 2) and the total number of days spanned by the observations (column 3 ). RVs (column 4) were either taken from Blake et al. (2010) or determined by cross-correlation of the highest signal-to-noise spectrum for a particular object with spectra of objects with known RV (from Blake et al.) and similar spectral type, sometimes of type both earlier and later than our target. The RVs resulting from crosscorrelation of a target with more than one other object were averaged and the standard deviation added in quadrature with the internal uncertainties in the RV measurements. This result in most cases was dominated by the $2 \mathrm{~km} \mathrm{~s}^{-1}$ internal uncertainty; however, for a few objects, primarily the fainter and thus lower signal to noise late $\mathrm{T}$ dwarfs, this procedure resulted in an uncertainty of $3 \mathrm{~km} \mathrm{~s}^{-1}$ (Table 2).

We use the average RV values from Blake et al. (2010) when available because of their unprecedented precision, obtained by fitting models to near-IR K-band $\mathrm{CO}$ bandhead at $\sim 2.3 \mu \mathrm{m}$ target spectra. The models are composed of synthetic template spectra plus observed telluric spectra; the $\mathrm{CO}$ bandhead region of the telluric spectrum is rich in deep $\mathrm{CH}_{4}$ lines that provide a wavelength dispersion and zero-point reference with a precision as good as a few tens of $\mathrm{m} \mathrm{s}^{-1}$. Small iterations of the RV shift between the synthetic photospheric spectra and the telluric spectra allow for high accuracy in the target RV measurements. We compared our results with values from other RV studies in the literature, e.g., Basri et al. (2000), Mohanty \& Basri (2003), and Zapatero Osorio et al. (2007). In every case our RVs were comparable to other values within 1 $\sigma$. We provide our results where indicated in Table 2 .

\subsection{Rotational Velocities}

Column 5 of Table 2 lists the $v$ sin $i$ values for our targets. Most of these were taken from the literature (Basri et al. 2000; Mohanty \& Basri 2003; Rice et al. 2010; Blake et al. 2010 references given in column 6). To estimate $v \sin i$ values for the remaining targets, we used visual comparison with objects of neighboring spectral types after superimposing the spectra. For some objects we convolved comparison spectra of known $v \sin i$ with a boxcar kernel in order to produce resulting spectra of larger $v \sin i$ for comparison. This method was approximate and yielded uncertainties of $5-10 \mathrm{~km} \mathrm{~s}^{-1}$, based on visual comparisons with objects of known $v \sin i$, for the $\mathrm{T}$ dwarfs in our sample. Nevertheless, these are the first estimates available for some of the targets and thus provide a useful guide.

\section{DISCUSSION}

\subsection{Field Brown Dwarf Spectroscopic Multiplicity}

Konopacky et al. (2010) obtained angularly resolved imaging and spectroscopy for each component in 24 very low mass stellar and brown dwarf subarcsecond visual binaries contributing to eventual measurements of orbital solutions and component masses. Our goal was to use high spectral resolution observations to identify any RV variability over time that might indicate a spectroscopic binary brown dwarf. This requires binaries with orbital periods sufficiently short to measure the component motion at a significant level, i.e., at least several $\sigma$ above the RV uncertainty. 
Table 2

Radial and Rotational Velocities

\begin{tabular}{|c|c|c|c|c|c|}
\hline $\begin{array}{l}\text { Object } \\
\text { (1) }\end{array}$ & $\begin{array}{c}N \\
\text { Obs. } \\
(2)\end{array}$ & $\begin{array}{c}\Delta T \\
\text { (days) } \\
(3)\end{array}$ & $\begin{array}{c}\langle R V\rangle \\
\left(\mathrm{km} \mathrm{s}^{-1}\right) \\
(4)\end{array}$ & $\begin{array}{c}v \sin i \\
\left(\mathrm{~km} \mathrm{~s}^{-1}\right) \\
(5)\end{array}$ & $\begin{array}{l}\text { References } \\
\langle R V\rangle, v \sin i \\
(6)\end{array}$ \\
\hline GJ 406 & 3 & 1488 & $19 \pm 2$ & $\leqslant 8 \pm 2$ & Rice et al. (2010) \\
\hline $2 \mathrm{M} 0140+27$ & 3 & 566 & $9 \pm 2$ & $11 \pm 2$ & Rice et al. (2010) \\
\hline 2M0345+25 & 3 & 1864 & $6 \pm 3$ & $30 \pm 5$ & This work \\
\hline $2 \mathrm{M} 0746+20^{\mathrm{a}}$ & 2 & 1470 & $52.37 \pm 0.06$ & $32.72 \pm 0.56$ & Blake et al. (2010) \\
\hline 2M2057-02 & 3 & 499 & $-24.68 \pm 0.43$ & $60.56 \pm 0.37$ & Blake et al. (2010) \\
\hline $2 \mathrm{M} 0015+35$ & 2 & 3054 & $-37.35 \pm 0.16$ & $10.23 \pm 2.55$ & Blake et al. (2010) \\
\hline Kelu- $1^{\text {a }}$ & 7 & 3214 & $6.37 \pm 0.35$ & $68.88 \pm 2.60$ & Blake et al. (2010) \\
\hline 2M2104-10 & 2 & 893 & $-21.09 \pm 0.12$ & $23.44 \pm 0.23$ & Blake et al. (2010) \\
\hline G196-3B & 4 & 2159 & $-2 \pm 2$ & $10 \pm 2$ & This work, Mohanty \& Basri (2003) \\
\hline 2M1507-16 & 5 & 2887 & $-39.85 \pm 0.05$ & $21.27 \pm 1.86$ & Blake et al. (2010) \\
\hline DENIS $0205^{\mathrm{a}}$ & 3 & 1554 & $7 \pm 2$ & $22 \pm 5$ & This work, Basri et al. (2000) \\
\hline DENIS $1228^{\mathrm{a}}$ & 3 & 1347 & $2 \pm 2$ & $22 \pm 3$ & This work, Basri et al. (2000) \\
\hline SDSS $0423-04^{\mathrm{a}}$ & 3 & 1554 & $28 \pm 2$ & $60 \pm 10$ & This work \\
\hline SDSS 1254-01 & 2 & 1478 & $4 \pm 3$ & $15 \pm 5$ & This work \\
\hline $2 \mathrm{M} 2254+31$ & 2 & 709 & $14 \pm 3$ & $15 \pm 5$ & This work \\
\hline 2M0559-14 & 4 & 2353 & $-9 \pm 3$ & $10 \pm 5$ & This work \\
\hline 2M2356-15 & 2 & 144 & $19 \pm 3$ & $15 \pm 5$ & This work \\
\hline $2 \mathrm{M} 0937+29$ & 5 & 1487 & $-5 \pm 3$ & $60 \pm 10$ & This work \\
\hline
\end{tabular}

Note.

a Binary system.

To explore our sensitivity to the brown dwarf binary parameter space, given our $\sim 2 \mathrm{~km} \mathrm{~s}^{-1} \mathrm{RV}$ precision, we ran a Monte Carlo simulation of $10^{5}$ possible binary orbits for each of the 25 objects in our sample, following Burgasser et al. (2014). Orbital parameters were uniformly distributed in log semimajor axis $\left(10^{-3}-10^{2} \mathrm{AU}\right)$, mass ratio $(0.8-1.0)$, sine inclination (0-1), eccentricity (0-0.6; Dupuy \& Liu 2011), and all other orbital parameters (argument of periapsis, longitude of ascending node, and mean anomaly, $0-2 \pi$ ). We converted our target spectral types to effective temperature using the empirical relation of Stephens et al. (2009), and then from effective temperature to mass using the evolutionary models of Burrows et al. (2001), assuming ages of 0.5, 1.0, and 5 Gyr. Each of the $10^{5}$ simulated orbits was sampled at the dates given and the primary orbital RV was calculated. A binary detection, for a given semimajor axis bin $(0.2 \mathrm{dex})$, was defined as a system for which a maximum RV difference between all dates was $>3 \sigma$, i.e., $>6 \mathrm{~km} \mathrm{~s}^{-1}$ given our $2 \mathrm{~km} \mathrm{~s}^{-1}$ precision. The results are summarized in Figure 5. The most important factor impacting the probability of detecting a potential binary was the frequency of observation for a given target (Table 2).

A binary with a separation of $\lesssim 0.1$ AU should in principle be straightforward to detect with an RV precision of $2 \mathrm{~km} \mathrm{~s}^{-1}$. However, given our estimated target masses and sampling frequency, and assuming an age of $1.0 \mathrm{Gyr}$, we could have detected such an orbit only $50 \%$ of the time for only 12 of the sources in our sample (middle left-hand panel of Figure 5). The detection probability for an 0.1 AU binary fails to reach $90 \%$ for any of our sources. Using the probabilities of detection for

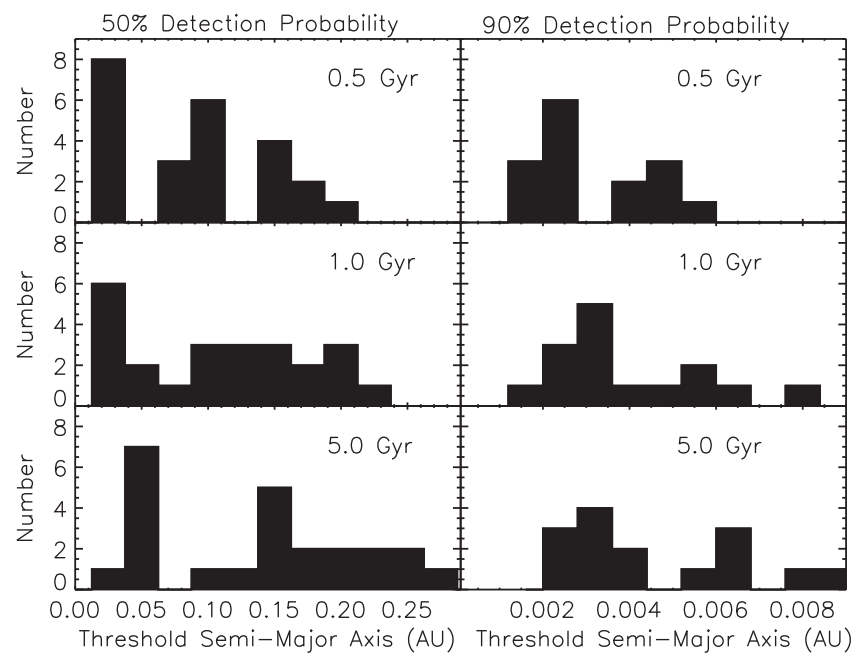

Figure 5. Results from our Monte Carlo simulation showing the number of our targets with 50\% (left panels) and 90\% (right panels) spectroscopic binary detection probability as a function of the threshold semimajor axis (Section 4.3), given our target properties and RV precision. Because mass is a function of age, we present results for three age bins; spectral types were converted to mass estimates using models and empirical relations (see the text). The outliers Kelu-1 and 2M0036, more favorable to detection because of the combination of their relatively high mass and our high sampling frequency, are not included in the right hand panels but ranged between 0.011 and $0.015 \mathrm{AU}$ for a $90 \%$ detection probability. Kelu- 1 is not included in the left panels, either, but its detection probabilities for the three ages ranged from 0.34 to $0.47 \mathrm{AU}$ for a $50 \%$ detection probability. In addition to Kelu- 1 and 2M0036, only 15/25 objects had $90 \%$ detection probabilities, thus fewer objects are plotted in the right panels. Bin sizes are 0.025 AU on the left and 0.0008 AU on the right. 


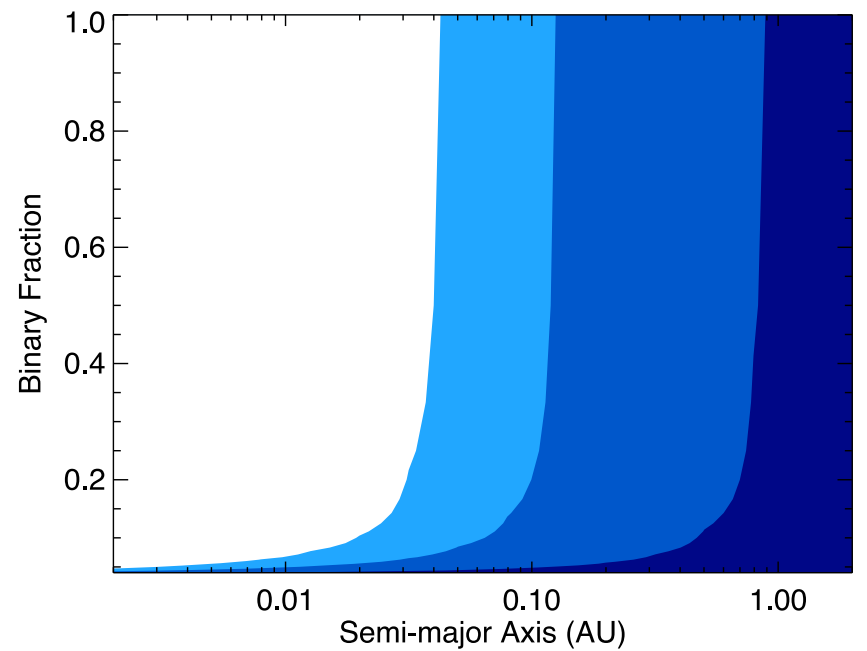

Figure 6. Binary fraction detection thresholds as a function of the typical semimajor axis based on the velocity dispersion of our sample. Colored contours correspond to velocity dispersions 1.5, 3, and 5 times higher (from right to left) than the observed dispersion $\sigma$, computed by sampling randomly oriented orbits with the given semimajor axis at the observational epochs of our sample. The most conservative threshold $(5 \sigma)$ corresponds to semimajor axes of 0.03-0.04 AU.

separations greater than a given threshold $a, P(>a)$, as a measure of the effective sample size, $N_{\mathrm{eff}}(a)=\Sigma_{i} P(>a)$, we find our null result translates into a $1 \sigma$ upper limit of $18 \%$ for spectroscopic binaries down to $a=0.1 \mathrm{AU}$, based on binomial statistics. Only for systems with separations below $0.01 \mathrm{AU}$ ( $\sim 1$ day orbits) could the spectroscopic binary frequency of our sample be characterized as relatively rare, i.e., $\lesssim 10 \%$.

These limits apply when we consider the detectability of individual systems. However, a signature of unresolved multiplicity could also emerge in higher velocity dispersions for the sample as a whole. Identifying higher dispersions across the sample requires robust determination of the individual measurement uncertainties, but we can perform a rough assessment as follows. Using the same simulation parameters, we calculated the distribution of velocity dispersions one would obtain if a given fraction of sources (randomly selected) were binaries with semimajor axes in logarithmically spaced bins. For a sample devoid of binaries, the mean dispersion is somewhat less than the adopted measurement uncertainty, about $1.75 \mathrm{~km} \mathrm{~s}^{-1}$. Sources with radial orbital motion drive the mean velocity dispersions of the sample higher. Figure 6 displays the thresholds at which the mean simulated sample velocity dispersions are $1.5,3$, and 5 times higher than the dispersions assuming a $2 \mathrm{~km} \mathrm{~s}^{-1}$ measurement uncertainty. The most conservative threshold is reached at a semimajor axis of 0.03-0.04 AU, and is detectable at even small binary fractions (i.e., 1-2 sources in the sample being binary). This analysis is roughly consistent with the individual detection limits above, and again implies that we can rule out a significant fraction of binaries $(\gtrsim 10 \%)$ only for separations $\lesssim 0.01 \mathrm{AU}$.

\subsection{Notes on Known Visual Binaries}

Of the 25 targets in our sample, 7 are known visual binaries. For these systems we estimated the upper limit for the observable $\mathrm{RV}$ shift, $\Delta(\mathrm{RV})_{\max }$, for the brighter binary component between two epochs, assuming the most favorable possible observing conditions: (1) the epochs correspond to the two phases at which the primary component is moving toward and away from us with maximum RV, (2) the projected separation corresponds to the semimajor axis of the system, and (3) the orbit is circular and edge-on. The observed and estimated binary properties are given in Table 3. A discussion of each visual binary and the results of our observations follows.

\subsubsection{MASS J22344161+4041387-M6}

Using laser guide star adaptive optics imaging at the Keck II telescope, Allers et al. (2009) identified 2M2234 as a $1 \mathrm{Myr}$ year old, visual binary with a projected physical separation of 51 AU. Given the observed binary properties, the $\Delta(\mathrm{RV})_{\max }$ is $\sim 1.9 \mathrm{~km} \mathrm{~s}^{-1}$ (Table 3 ). With an orbital period of $824_{-310}^{+510}$ years the inclination is effectively indeterminable. This estimate for the period is based on a circular orbit. A more realistic value, $1000_{-500}^{+1600}$ years, is calculated in Allers et al. (2009). In either case, it is not possible to observe the system at phases separated by half the orbit. Furthermore, given the $v \sin i$ of $17 \mathrm{~km} \mathrm{~s}^{-1}$ for 2M2234 (Table 2), it is also impossible to spectroscopically resolve the RVs of the two components in a single epoch spectrum, even though the component near-IR magnitudes are almost equal, because the maximum relative component RV separation is significantly less than the rotational broadening.

Cross-correlation of our 3 epochs of spectra with each other demonstrated no RV shift between the 2007 and 2009 data. Between the 2006 and 2007 data there was an apparent shift of $-7.7 \mathrm{~km} \mathrm{~s}^{-1}$; however, the signal to noise of the 2006 spectrum $(\sim 20)$ is considerably lower than that of the other epochs $(\sim 80)$, and the spectra are veiled (Allers et al. 2009), thus we do not have confidence in the 2006 result. Using the young M6 brown dwarf [GY92] 5 for cross-correlation with our 2007 spectrum, we obtain an RV of $-10 \pm 2 \mathrm{~km} \mathrm{~s}^{-1}$ (Table 2), ${ }^{11}$ similar to the results of Allers et al. on the basis of Keck HIRES data from $2006,{ }^{12}-10.6 \pm 0.5 \mathrm{~km} \mathrm{~s}^{-1}$. Allers et al. raise the possibility that $2 \mathrm{M} 2234$ could be a higher order multiple system, which would account for the overluminous nature of the A component. Our multi-epoch observations failed to detect any short-period, i.e., $P<a$ few years, hierarchical spectroscopic binary in this system, although our sensitivity to intermediate separation binaries, and binary orientations unfavorable for detection, limit any significant statistical conclusions (Section 4.1). Given the greater $K_{s}-L^{\prime}$ excess in the 2M2234A, it is feasible that the excess luminosity is related to the circumstellar disk structure, orientation, and/or possible accretion activity. Such a mismatch in disk properties around the components of very low mass binaries is not unprecedented; for example, the TWA $30 \mathrm{AB}$ wide, co-moving pair has an apparently edge-on disk around the embedded, earlier-type component, extinguishing this late type $M$ star by 5 magnitudes with respect to the cooler component (Looper et al. 2010).

\subsubsection{MASS J07464256+2000321-L1}

The 2 M0746 binary is a nearby $(d \sim 12 \mathrm{pc})$, tight $(\sim 3 \mathrm{AU})$ system. We use the Konopacky et al. (2010) astrometric

\footnotetext{
${ }^{11}$ Cross-correlating the same 2M2234 spectrum with another young M6, CFHT Tau 7, Rice et al. (2010) found $-13.1 \mathrm{~km} \mathrm{~s}^{-1}$.

${ }^{12}$ This result is the weighted mean of two RVs; Shkolnik et al. (2012) use the same Keck measurements to determine an unweighted mean of $-10.9 \pm$ $0.7 \mathrm{~km} \mathrm{~s}^{-1}$.
} 
Table 3

Observed and Estimated Binary Properties

\begin{tabular}{|c|c|c|c|c|c|c|c|}
\hline $\begin{array}{l}\text { Object } \\
(1)\end{array}$ & $\begin{array}{l}M_{\text {total }} \\
\left(M_{\odot}\right) \\
(2)\end{array}$ & $\begin{array}{l}\text { Distance } \\
(\mathrm{pc}) \\
(3)\end{array}$ & $\begin{array}{l}\text { Separation } \\
\left({ }^{\prime \prime}\right) \\
(4)\end{array}$ & $\begin{array}{c}\text { Semimajor Axis }^{\mathrm{a}} \\
(\mathrm{AU}) \\
(5)\end{array}$ & $\begin{array}{l}\text { Period }^{\text {a }} \\
\text { (years) } \\
(6)\end{array}$ & $\begin{array}{c}\Delta(\mathrm{RV})_{\max }{ }^{\mathrm{a}} \\
\left(\mathrm{km} \mathrm{s}^{-1}\right) \\
(7)\end{array}$ & $\begin{array}{l}\text { References } \\
(8)\end{array}$ \\
\hline $2 \mathrm{M} 2234+40$ & $0.20_{-0.06}^{+0.11}$ & $325_{-50}^{+72}$ & $0.1582 \pm 0.0003$ & $51_{-8}^{+12}$ & $824_{-310}^{+510}$ & $1.9_{-0.5}^{+0.6}$ & Allers et al. (2009) \\
\hline $2 \mathrm{M} 0746+20^{\mathrm{b}}$ & $0.151 \pm 0.003$ & $12.21 \pm 0.05^{\mathrm{d}}$ & $0.2373_{-0.0040}^{+0.0015 c}$ & $2.90_{-0.06}^{+0.03}$ & $12.71 \pm 0.07$ & $6.8 \pm 0.1^{\mathrm{e}}$ & Konopacky et al. (2010) \\
\hline Kelu-1 & $0.115 \pm 0.014$ & $18.7 \pm 0.7^{\mathrm{d}}$ & $0.298 \pm 0.003$ & $5.6 \pm 0.3$ & $39 \pm 5$ & $4.3 \pm 0.4$ & Gelino et al. (2006) \\
\hline DENIS 0205 & 0.15 & $19.76 \pm 0.57^{\mathrm{d}}$ & $0.35^{\mathrm{f}}$ & 6.9 & 47 & 4.4 & Bouy et al. (2006) \\
\hline DENIS 1228 & 0.135 & $20.24 \pm 0.08^{\mathrm{d}}$ & $0.32^{\mathrm{c}}$ & 6.41 & 44.2 & 4.3 & Brandner et al. (2004) \\
\hline SDSS 0423-04 & $0.08-0.14$ & $15.2 \pm 0.4^{\mathrm{g}}$ & $0.1642 \pm 0.0017$ & $2.50 \pm 0.07$ & $10.5-13.9$ & $5.3-7.1$ & Burgasser et al. (2005) \\
\hline
\end{tabular}

Notes.

a Assuming eccentricity is 0 and inclination is $90^{\circ}$.

${ }^{\mathrm{b}}$ All parameters except for $\Delta(\mathrm{RV})_{\max }$ from Konopacky et al. (2010).

c Semimajor axis (").

d Dahn et al. (2002).

e Average $v_{\text {orbital }}$, modulo inclination of $138^{\circ}$; consistent with Konopacky et al. (2010) fit to $K_{1}+K_{2}$.

${ }^{f}$ Average observed separation from Bouy et al. (2006).

g Vrba et al. (2004).

measurements (Table 3) to determine a $\Delta(\mathrm{RV})_{\max }$ of $2.0 \mathrm{~km} \mathrm{~s}^{-1}$. Konopacky et al. find an average primary/ secondary flux ratio of $1.5 \pm 0.1$, challenging the assumption that angularly unresolved spectra are fully dominated by the primary (Blake et al. 2010).

We observed 2M0746 at two epochs separated by almost exactly 4 years, about $1 / 3$ of the orbital period. Crosscorrelation of our two order $62 \mathrm{~J}$-band spectra yielded a $1.3 \mathrm{~km} \mathrm{~s}^{-1}$ shift with a high correlation coefficient, 0.92 . Comparing the epochs of our observations with the RV curve plotted for this system in Figure 14 of Blake et al. (2010), this is almost exactly the expected result; however, we are not sufficiently confident in our RV uncertainties to give it much weight.

\subsubsection{Kelu-1-L2}

A rapid rotator with $v$ sin $i$ of $\sim 70 \mathrm{~km} \mathrm{~s}^{-1}$ and $\mathrm{Li}$ absorption, Kelu-1 was identified as a brown dwarf by Ruiz et al. (1997). Martín et al. (1999) hypothesized that Kelu-1's over-luminosity and $\mathrm{Li}$ abundance might be explained by a young age or an additional component in the system (e.g., Golimowski et al. 2004). Liu \& Leggett (2005) using Keck AO imaging found that Kelu-1 was a $0{ }^{\prime \prime} .291$ binary. Gelino et al. (2006) estimated spectral types for the components of L2 and L3.5 and a total mass of $0.115 \pm 0.014 M_{\odot}$. In an unpublished preprint, Stumpf et al. (2008) describe additional observations of the system with VLT AO imaging through 2008; the separation steadily increased to $0^{\prime \prime} .366$ in 2008 . The position angle has not changed by more than $4^{\circ}$ or $5^{\circ}$. Adopting the largest separation observed by Gelino et al. as the semimajor axis, 0 ". $298 \pm 0$ ". 003 , we estimate a period of $39 \pm 5$ years based on a circular orbit (although Stumpf et al. favor a high eccentricity of $0.82 \pm 0.10$ ). If viewed edge-on, this implies a $\Delta(\mathrm{RV})_{\max }$ of $4.3 \pm 0.4 \mathrm{~km} \mathrm{~s}^{-1}$, marginally detectable with our $\sim 2 \mathrm{~km} \mathrm{~s}^{-1}$ precision.

Measurements of the Kelu-1 system RV in the literature are inconsistent: Basri et al. (2000) found $17 \pm 1 \mathrm{~km} \mathrm{~s}^{-1}$ in 1997 June and Blake et al. (2010) determined RVs of $6.35 \pm 0.39$ and $6.41 \pm 0.75 \mathrm{~km} \mathrm{~s}^{-1}$ in 2003 March and April. On the basis of angularly resolved spectra of the two known components, Stumpf et al. (2008) suggest that Kelu-1 A is itself a spectroscopic binary. We used our highest $\mathrm{S} / \mathrm{N}$ spectrum of Kelu-1 to cross-correlate against five other epochs (Figure 3), all of $\mathrm{S} / \mathrm{N}>50$ per resolution element (the 2006 January, spectrum, with a $\mathrm{S} / \mathrm{N}$ of $\sim 10$, was not included in this analysis). Our RV measurements, from 2002 through 2011, show RV shifts of $<3 \mathrm{~km} \mathrm{~s}^{-1}$. We did not detect any clear evidence in our spectra for additional motion resulting from the A-component moving in a relatively short-period spectroscopic orbit; however, this could conceivably be the result of binary properties and/or viewing geometry (Section 4.1).

\subsubsection{MASS J15074769-1627386-L5.5}

Over a 6 year baseline, Blake et al. (2010) detect a marginally significant $(<2 \sigma)$ trend in the RV of $2 \mathrm{M} 1507$, a nearby $(d=7.3 \mathrm{pc})$ L dwarf. They obtain a false alarm probability of $2.2 \%$ and suggest the possibility that $2 \mathrm{M} 1507$ is a >5000 day binary with an angular separation of 0 ". 4 . However, deep, high-resolution imaging sensitive to a contrast ratio of 5 mag (Bouy et al. 2003; Reid et al. 2006) has not revealed any companions. No significant RV variations are evident in the 5 high-resolution spectra we obtained between 2000 and 2008; cross-correlation of the highest S/N spectrum (UT 2000 April 25) against the other 4 epochs resulted in RV shifts of $<1.7 \mathrm{~km} \mathrm{~s}^{-1}$ with an uncertainty of $\sim 2 \mathrm{~km} \mathrm{~s}^{-1}$. This result, however, does not rule out multiplicity; Blake et al. observed $\sim 0.5 \mathrm{~km} \mathrm{~s}^{-1}$ of motion over 6.5 years, thus we would not expect much more than that over our 8 year baseline. Given the lack of definitive evidence for multiplicity, this system is not included in Table 3.

\subsubsection{DENIS-P J0205.4-1159—L5.5}

Koerner et al. (1999) initially identified this system as binary. Bouy et al. (2005) describe evidence for a third object in a bound orbit with the secondary component. The estimated properties of the wide binary orbit are uncertain but the period is at least 47 years and the $\Delta(\mathrm{RV})_{\max }$ is at most $4.4 \mathrm{~km} \mathrm{~s}^{-1}$ (Table 3). For the presumed close binary, Bouy et al. estimate an orbital period of 8 years and a semimajor axis of 1.9 AU, implying a $\Delta(\mathrm{RV})_{\max }$ of $\sim 7 \mathrm{~km} \mathrm{~s}^{-1}$. Our three spectra of DENIS 0205, taken in 2001 and in 2006, are of low S/N. Cross-correlation between the epochs yields -2.7 
and $-2.1 \mathrm{~km} \mathrm{~s}^{-1}$ with a correlation coefficient of only $\sim 0.4$, reflecting the poor quality of the data. Sufficiently frequent and deep imaging and RV monitoring of this system may provide the requisite phase coverage, preferably with better precision than $2 \mathrm{~km} \mathrm{~s}^{-1}$, to determine a full orbital solution for the inner binary over the course of one orbital period.

\subsubsection{DENIS-P J1228.2-1547—L6}

Using the Hubble Space Telescope, Martín et al. (1999) identified DENIS 1228 as the first angularly resolved brown dwarf-brown dwarf pair with a separation of 0 ". $275 \pm 0$ ".002 (Bouy et al. 2003). After several years of monitoring the components' positions, Brandner et al. (2004) estimated the orbital properties of the system, listed in Table 3 . The $\Delta$ $(\mathrm{RV})_{\max }$ for this binary, $4.3 \mathrm{~km} \mathrm{~s}^{-1}$, in combination with the period of $\sim 44$ years from Brandner et al., is not favorable for the detection of an RV shift over the 4 year timescale of our NIRSPEC observations. Cross-correlating our 2007 May spectrum with those taken in 2011 February and June yields a $0 \mathrm{~km} \mathrm{~s}^{-1} \mathrm{RV}$ shift. Continued monitoring of the visual orbit with high angular resolution imaging and high precision RV spectroscopic techniques will help to refine the parameters over the next decades, necessary to determine individual component masses in the long term.

\subsubsection{SDSSp J042348.57-041403.5-T0}

SDSS 0423 is one of the visual brown dwarf binary systems which spans the $\mathrm{L}$ and $\mathrm{T}$ classes. Burgasser et al. (2005) measured a separation of $0^{\prime \prime} .16$ and estimated a total mass of $0.08-0.14 M_{\odot}$. Assuming that the separation is equal to the semimajor axis of the system, $2.50 \pm 0.07 \mathrm{AU}$, the period falls in the range of 10.5-13.9 years (Table 3 ) and the $\Delta(\mathrm{RV})_{\max }$ is $5.3-7.1 \mathrm{~km} \mathrm{~s}^{-1}$. We observed the system in 2001, 2005, and 2006, covering close to half of the estimated orbital period. However, the cross-correlation between the 2001 and 2005 spectra yielded a shift of only $-0.4 \mathrm{~km} \mathrm{~s}^{-1}$ and between 2001 and 2006 of $1.77 \mathrm{~km} \mathrm{~s}^{-1}$, indistinguishable within the uncertainty of our RV measurements, especially because the 2006 spectrum was particularly noisy. Thus we find no evidence for significant orbital motion, implying a longer period or an unfavorable viewing geometry for the detection of an RV shift, or both.

\subsection{MASS J05591914-1404488}

The T4.5 dwarf 2M0559 presents an enigmatic case of an over-luminous, extremely low-mass object. Observers and theorists alike have speculated (Burgasser 2001; Dahn et al. 2002; Burrows et al. 2006; Dupuy \& Liu 2012) that this system is an equal mass binary. Alternatively, there may be fundamental processes at play in the mid-T dwarf atmospheres that are not yet well-understood. Specifically, this source is the lynchpin in the J-band brightening/cloud disruption scenario (Burgasser et al. 2002a). Zapatero Osorio et al. (2007) estimate limits on possible planetary mass companions in this system, but such a secondary component would not explain the unusually high brightness.

We obtained four observations of 2M0559 over a time baseline of 6.5 years. For an age of $1 \mathrm{Gyr}$, our Monte Carlo simulation (Section 4.1) indicates a $50 \%$ detection probability for a threshold semimajor axis of $0.13 \mathrm{AU}$ and a $90 \%$ detection probability for a threshold semimajor axis of $0.003 \mathrm{AU}$. The threshold semimajor axis is the separation below which a spectroscopic companion would be detected with a particular probability. Burgasser et al. (2003) rule out the presence of a relatively bright companion object closer than 0 ". 09 . At the $\sim 10$ pc distance to 2M0559 (Dahn et al. 2002), 0".09 corresponds to $\sim 0.9 \mathrm{AU}$. Thus, ample parameter space for a bright binary companion to this object remains unexplored and our confidence in a null result for a companion object is only high $(\geqslant 90 \%)$ for extremely short periods of days or less. Monitoring this system with extremely precise RV measurements (see next section) with regular cadence over a considerable time baseline will help to fill in the potential binary parameter space gap and might also provide insight into the atmospheric properties.

\subsection{The Importance of High Precision RV Measurements}

For spectroscopic binary systems, Figure 7 illustrates the relationships between the primary object's mass, the primary orbital velocity, and the orbital period on the basis of Kepler's third law. We show results for three distinct values of the mass ratio $(q)$; a circular, edge-on orbit is assumed for simplicity. For a system with a primary of mass 0.08 , the substellar limit, and a mass ratio of 1.0 , the primary object's RV is $\sim 3.5 \mathrm{~km} \mathrm{~s}^{-1}$ for a period of 12 years, approximately the shortest period system among the visual binaries in our sample (Table 3 ). With a precision of $2 \mathrm{~km} \mathrm{~s}^{-1}$, motion of the primary (or the secondary, given a mass ratio of unity) in such a system is only detectable for very specific phases and viewing angles. The probability of detection with $2 \mathrm{~km} \mathrm{~s}^{-1}$ precision increases for shorter-period binaries; however, again, this is only true under certain specialized conditions (Section 4.1). None of the multi-epoch spectra in our sample of 25 brown dwarf systems reveals more than $\sim 3 \mathrm{~km} \mathrm{~s}^{-1}$ of $\mathrm{RV}$ variability. Even for the seven known brown dwarf binaries observed, some with a cadence that regularly sampled a significant fraction of the estimated orbital period, we were unable to unambiguously detect any RV variability.

Specialized techniques for the highly precise measurement of small RV shifts, such as those applied to high-resolution K-band spectra by Blake et al. (2007, 2010), Prato et al. (2008), Konopacky et al. (2010), Bailey et al. (2012), Burgasser et al. (2012), and others, are required to reliably detect motion in brown dwarf binaries, even for those with orbital periods as short as days. In their 6 year study of latetype $M$ and L dwarfs with NIRSPEC on the Keck II telescope, Blake et al. (2010) obtained a precision of $200 \mathrm{~m} \mathrm{~s}^{-1}$ on slowly rotating $\mathrm{L}$ dwarfs, providing sensitivity to orbital motion of brown dwarf binaries with periods of decades and mass ratios as low as $\sim 10 \%$ (Figure 7 ), the upper limit for the detection of giant planetary companions.

In the study described here, even for our sample of 25 systems with zero detections of spectroscopic binaries, it is still not possible to use the results to definitively characterize shortperiod low mass binaries as rare. The sampling and geometry of such systems are simply not well-suited to identification with our $2 \mathrm{~km} \mathrm{~s}^{-1}$ precision and random observing cadence. Thus as far as it is possible to say with the extant data, very low mass spectroscopic binaries are not necessarily intrinsically rare, but even with one of the largest samples available, statistics show (Section 4.1 ) that $2 \mathrm{~km} \mathrm{~s}^{-1}$ uncertainties provide relatively weak constraints. 

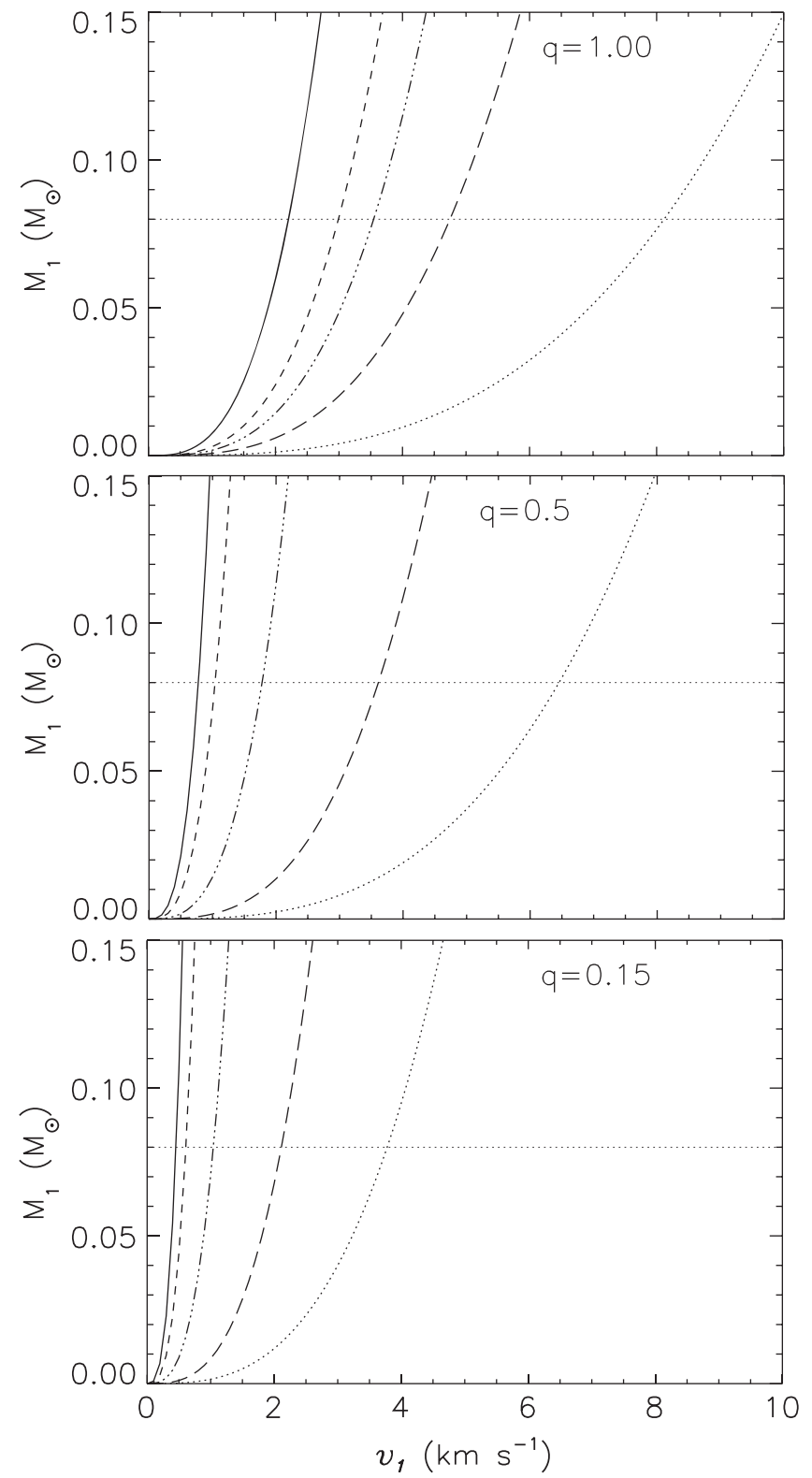

Figure 7. Each panel illustrates the relationship in a spectroscopic binary between primary object mass $\left(M_{1}\right)$, primary orbital velocity $\left(v_{1}\right)$, and orbital period, curved lines, for three sample mass ratios, $q=1,0.5$, and 0.15 , top to bottom. Plots for periods of 50 years (solid line), 20 years (short dash line), 12 years (dashed-dotted line), 5 years (long dash line), and 1 year (dotted line) are shown. The horizontal dotted line demarcates the nominal sub-stellar cut off for the more massive star in the system. The orbits are assumed to be circular and edge-on, the most favorable possible orientation for detection.

\section{SUMMARY}

We obtained multiple-epoch spectra of a sample of 25 very low-mass field dwarfs, $3 \mathrm{M}$ dwarfs, $16 \mathrm{~L}$ dwarfs, and $6 \mathrm{~T}$ dwarfs, between 2000 April and 2011 June to search for RV variability and spectral evidence for multiple components. With a precision of $\sim 2 \mathrm{~km} \mathrm{~s}^{-1}$, we were sensitive to RV variability at a statistically significant level only in systems with periods of about a day or less, assuming a favorable distribution of orbital properties and viewing geometries relative to our line of sight. In none of the systems studied, including the seven known, wide binaries observed, did we detect any RV variability $>3 \mathrm{~km} \mathrm{~s}^{-1}$. For over a dozen objects in our sample we present the first published high-resolution spectra and provide RVs and rotational velocities for the entire sample, either based on this work or taken from the more precise measurements in Blake et al. (2010). We show multi-epoch spectral sequences for two objects of particular interest, Kelu-1 and 2M0937, an L2 and a peculiar T6, respectively. No significant variations are seen in these or the other target spectra, some of which boast an exquisite $\mathrm{S} / \mathrm{N}$ in excess of 100 .

$\mathrm{RV}$ measurements of brown dwarfs are important both for the ultimate measurement of brown dwarf masses (Konopacky et al. 2010) and for the spectroscopic detection of very lowmass, even planetary, companions to presumed single brown dwarfs (Blake et al. 2010). The close binary fraction of very low mass systems is highly uncertain (e.g., Bardalez Gagliuffi et al. 2014). We conclude with the observation that to satisfy these scientific goals requires a high $\mathrm{S} / \mathrm{N}$, strategic sampling cadence, and relatively high precision measurements: with the $200 \mathrm{~m} \mathrm{~s}^{-1}$ precision of Blake et al., it is possible to detect several-Jupiter mass companions even in orbits of decades (bottom panel, Figure 7). Long-term monitoring programs of binary brown dwarfs, and in particular candidate spectroscopic binary brown dwarfs (Bardalez Gagliuffi et al.), with high spectral resolution, component-resolved spectroscopy (Konopacky et al.), with high spectral resolution unresolved spectroscopy (Burgasser et al. 2012), and with high-angular resolution imaging (e.g., Radigan et al. 2013), over timescales of days to years are required. Results of these efforts will yield component mass measurements with sufficient precision to stringently test models of brown dwarf structure and evolution, and, in the case of younger systems, formation (e.g., Schaefer et al. 2014). It is crucial that RV monitoring programs take advantage of highprecision techniques for a future high-yield science return.

We thank the Keck Observatory OAs and SAs and B. Schaefer, probably all of whom helped with these runs and observations during the 11 year period over which the data were gathered, for their exceptional support. We are grateful to Q. Konopacky and M. McGovern for assistance with some of the later observing runs. L.P. thanks O. Franz and L. Wasserman for helpful discussions on orbital dynamics. We are grateful to the anonymous referee for comments that improved this manuscript. Partial support to L.P. for this work was provided by NSF grant AST 04-44017. This research has benefited from the M, L, T, and Y dwarf compendium housed at DwarfArchives.org. This work made use of the SIMBAD reference database, the NASA Astrophysics Data System, and the data products from the 2MASS, which is a joint project of the University of Massachusetts and the Infrared Processing and Analysis Center/California Institute of Technology, funded by the National Aeronautics and Space Administration and the National Science Foundation. Data presented herein were obtained at the W. M. Keck Observatory, which is operated as a scientific partnership among the California Institute of Technology, the University of California, and the National Aeronautics and Space Administration. The Observatory was made possible by the generous financial support of the W. M. Keck Foundation. The authors recognize and acknowledge the significant cultural role that the summit of Mauna Kea plays within the indigenous Hawaiian community. We are grateful for the opportunity to conduct observations from this special mountain. 


\section{REFERENCES}

Abt, H. A., \& Levy, S. G. 1976, ApJS, 30, 273

Allers, K. N., Liu, M. C., Shkolnik, E., et al. 2009, ApJ, 697, 824

Armitage, P. J., \& Bonnell, I. A. 2002, MNRAS, 330, L11

Artigau, É., Bouchard, S., Doyon, R., \& Lafreniére, D. 2009, ApJ, 701, 1534

Bailey, J. I., III, White, R. J., Blake, C. H., et al. 2012, ApJ, 749, 16

Bakos, G. Á, Howard, A. W., Noyes, R. W., et al. 2009, ApJ, 707, 446

Bardalez Gagliuffi, D. C., Burgasser, A. J., Gelino, C. R., et al. 2014, ApJ, 794, 143

Basri, G., \& Martín, E. L. 1999, AJ, 118, 2460

Basri, G., Mohanty, S., Allard, F., et al. 2000, ApJ, 538, 363

Basri, G., \& Reiners, A. 2006, AJ, 132, 663

Becklin, E. E., \& Zuckerman, B. 1988, Natur, 336, 656

Bennett, D. P., Bond, I. A., Udalski, A., et al. 2008, ApJ, 684, 663

Blake, C. H., Charbonneau, D., White, R. J., Marley, M. S., \& Saumon, D. 2007, ApJ, 666, 1198

Blake, C. H., Charbonneau, D., White, R. J., et al. 2008, ApJL, 678, L125

Blake, C. H., Charbonneau, D., \& White, R. J. 2010, ApJ, 723, 684

Bouy, H., Brandner, W., Martín, E. L., et al. 2003, AJ, 126, 1526

Bouy, H., Martín, E. L., Brandner, W., \& Bouvier, J. 2005, AJ, 129, 511

Bouy, H., Martín, E. L., Brandner, W., et al. 2006, A\&A, 451, 177

Brandner, W., Martín, E. L., Bouy, H., et al. 2004, A\&A, 428, 205

Burgasser, A. J. 2001, PhD thesis, California Institute of Technology

Burgasser, A. J., Gillon, M., Melis, C., et al. 2014, arXiv:1410.4288

Burgasser, A. J., Kirkpatrick, J. D., Brown, M. E., et al. 2002b, ApJ, 564, 421

Burgasser, A. J., Kirkpatrick, J. D., Reid, I. N., et al. 2003, ApJ, 586, 512

Burgasser, A. J., Liu, M. C., Ireland, M. J., Cruz, K. L., \& Dupuy, T. J. 2008, ApJ, 681, 579

Burgasser, A. J., Luk, C., Dhital, S., et al. 2012, ApJ, 757, 110

Burgasser, A. J., Marley, M. S., Ackerman, A. S., et al. 2002a, ApJL, 571, L151

Burgasser, A. J., Reid, I. N., Leggett, S. K., et al. 2005, ApJL, 634, L177

Burgasser, A. J., Wilson, J. C., Kirkpatrick, J. D., et al. 2000, AJ, 120, 1100

Burrows, A., Hubbard, W. B., Lunine, J. I., \& Liebert, J. 2001, RvMP, 73, 719

Burrows, A., Sudarsky, D., \& Hubeny, I. 2006, ApJ, 640, 1063

Crockett, C. J., Mahmud, N. I., Prato, L., et al. 2012, ApJ, 761, 164

Cruz, K. L., Kirkpatrick, J. D., \& Burgasser, A. J. 2009, AJ, 137, 3345

Cruz, K. L., Reid, I. N., Liebert, J., Kirkpatrick, J. D., \& Lowrance, P. J. 2003, AJ, 126, 2421

Dahn, C. C., Harris, H. C., Levine, S. E., et al. 2008, ApJ, 686, 548

Dahn, C. C., Harris, H. C., Vrba, F. J., et al. 2002, AJ, 124, 1170

Delfosse, X., Tinney, C. G., Forveille, T., et al. 1997, A\&A, 327, L25

Dupuy, T. J., \& Liu, M. C. 2011, ApJ, 733, 122

Dupuy, T. J., \& Liu, M. C. 2012, ApJS, 201, 19

Dupuy, T. J., Liu, M. C., Bowler, B. P., et al. 2010, ApJ, 721, 1725

Duquennoy, A., \& Mayor, M. 1991, A\&A, 248, 485

Geballe, T. R., Knapp, G. R., Leggett, S. K., et al. 2002, ApJ, 564, 466

Gelino, C. R., Kulkarni, S. R., \& Stephens, D. C. 2006, PASP, 118, 611

Gizis, J. E., Monet, D. G., Reid, I. N., et al. 2000, AJ, 120, 1085

Golimowski, D. A., Henry, T. J., Krist, J. E., et al. 2004, AJ, 128, 1733

Grether, D., \& Lineweaver, C. H. 2006, ApJ, 640, 1051
Joergens, V. 2008, A\&A, 492, 545

Joergens, V., Bonnefoy, M., Liu, Y., et al. 2013, A\&A, 558, LL7

Joergens, V., Müller, A., \& Reffert, S. 2010, A\&A, 521, A24

Kirkpatrick, J. D., Beichman, C. A., \& Skrutskie, M. F. 1997, ApJ, 476, 311

Kirkpatrick, J. D., Reid, I. N., Liebert, J., et al. 2000, AJ, 120, 447

Koerner, D. W., Kirkpatrick, J. D., McElwain, M. W., \& Bonaventura, N. R. 1999, ApJL, 526, L25

Konopacky, Q. M., Ghez, A. M., Barman, T. S., et al. 2010, ApJ, 711, 1087

Leggett, S. K., Geballe, T. R., Fan, X., et al. 2000, ApJL, 536, L35

Liu, M. C., \& Leggett, S. K. 2005, ApJ, 634, 616

Lodieu, N., Pinfield, D. J., Leggett, S. K., et al. 2007, MNRAS, 379, 1423

Looper, D. L., Bochanski, J. J., Burgasser, A. J., et al. 2010, AJ, 140, 1486

Luhman, K. L., Adame, L., D’Alessio, P., et al. 2005, ApJL, 635, L93

Marois, C., Zuckerman, B., Konopacky, Q. M., Macintosh, B., \& Barman, T. 2010, Natur, 468, 1080

Martín, E. L., Delfosse, X., Basri, G., et al. 1999, AJ, 118, 2466

Mazeh, T., Simon, M., Prato, L., Markus, B., \& Zucker, S. 2003, ApJ, 599, 1344

McGovern, M. R., Kirkpatrick, J. D., McLean, I. S., et al. 2004, ApJ, 600, 1020

McLean, I. S., Becklin, E. E., Bendiksen, O., et al. 1998, Proc. SPIE, 566, 3354

McLean, I. S., Graham, J. R., Becklin, E. E., et al. 2000, Proc. SPIE, 4008, 1048

McLean, I. S., McGovern, M. R., Burgasser, A. J., et al. 2003, ApJ, 596, 561

McLean, I. S., Prato, L., Kim, S. S., et al. 2001, ApJL, 561, L115

McLean, I. S., Prato, L., McGovern, M. R., et al. 2007, ApJ, 658, 1217

Metchev, S. A., \& Hillenbrand, L. A. 2009, ApJS, 181, 62

Mohanty, S., \& Basri, G. 2003, ApJ, 583, 451

Prato, L., Huerta, M., Johns-Krull, C. M., et al. 2008, ApJL, 687, L103

Radigan, J., Jayawardhana, R., Lafrenière, D., et al. 2013, ApJ, 778, 36

Raghavan, D., McAlister, H. A., Henry, T. J., et al. 2010, ApJS, 190, 1

Rebolo, R., Zapatero Osorio, M. R., Madruga, S., et al. 1998, Sci, 282, 1309

Reid, I. N., Kirkpatrick, J. D., Gizis, J. E., et al. 2000, AJ, 119, 369

Reid, I. N., Lewitus, E., Allen, P. R., Cruz, K. L., \& Burgasser, A. J. 2006, AJ, 132,891

Reipurth, B., \& Clarke, C. 2001, AJ, 122, 432

Rice, E. L., Barman, T., Mclean, I. S., Prato, L., \& Kirkpatrick, J. D. 2010, ApJS, 186, 63

Ruiz, M. T., Leggett, S. K., \& Allard, F. 1997, ApJL, 491, L107

Sahlmann, J., Lazorenko, P. F., Ségransan, D., et al. 2013, A\&A, 556, A133

Schaefer, G. H., Prato, L., Simon, M., \& Patience, J. 2014, AJ, 147, 157

Shkolnik, E. L., Anglada-Escudé, G., Liu, M. C., et al. 2012, ApJ, 758, 56

Siegler, N., Close, L. M., Mamajek, E. E., \& Freed, M. 2003, ApJ, 598, 1265

Simon, M., Bender, C., \& Prato, L. 2006, ApJ, 644, 1183

Stassun, K. G., Mathieu, R. D., \& Valenti, J. A. 2006, Natur, 440, 311

Stephens, D. C., Leggett, S. K., Cushing, M. C., et al. 2009, ApJ, 702, 154

Stumpf, M. B., Brandner, W., Henning, T., et al. 2008, arXiv:0811.0556

Vrba, F. J., Henden, A. A., Luginbuhl, C. B., et al. 2004, AJ, 127, 2948

Wolf, M. 1919, VeHei, 7, 195

Zapatero Osorio, M. R., Lane, B. F., Pavlenko, Y., et al. 2004, ApJ, 615, 958

Zapatero Osorio, M. R., Martín, E. L., Béjar, V. J. S., et al. 2007, ApJ, 666,1205 